\title{
Postoperative Massive Pulmonary Embolism Due to Superficial Vein Thrombosis of the Upper Limb
}

\author{
Marco Cascella $^{\text {a, b }}$, Daniela Viscardi ${ }^{a}$, Francesca Bifulco ${ }^{\mathrm{a}}$, Arturo Cuomo ${ }^{\mathrm{a}}$
}

\begin{abstract}
It is well known that deep vein thrombosis of the upper extremities is linked to high morbidity/mortality, resulting in $12-20 \%$ of all documented pulmonary embolism; however, there are few data about thromboembolism originating from a vein and/or a branch of a superficial vein of the upper extremities. Pulmonary embolism secondary to upper limb superficial vein thrombosis (not combined with upper extremities deep vein thrombosis) is a very rare clinical manifestation with few cases reported in the literature. We report a rare case of thrombophlebitis in departure from a superficial branch of the cephalic vein of the right arm, complicated by cardiac arrest secondary to a massive pulmonary embolism in a patient who underwent major surgery for ovarian cancer. We discuss on the numerous thrombotic risk factors, triggering a cascade of reactions and resulting in a potential fatal clinical manifestation.
\end{abstract}

Keywords: Superficial vein; Thrombosis; Upper extremities; Deep venous thrombosis; Pulmonary embolism; Pulseless electrical activity

\section{Introduction}

It is well known that deep vein thrombosis of the upper extremities (UEDVT) is linked to high morbidity/mortality, resulting in $12-20 \%$ of all documented pulmonary embolism (PE) $[1,2]$; however, there are few data about thromboembolism originating from a vein and/or a branch of a superficial vein of the upper extremities.

The true incidence of superficial vein thrombosis (SVT) is underestimated because many cases remain undiagnosed. Although several cases of PE following an SVT of lower limb

Manuscript accepted for publication October 12, 2015

aDivision of Anesthesiology, Department of Anesthesiology, Endoscopy and Cardiology, Istituto Nazionale Tumori Fondazione Pascale, Naples, Italy

${ }^{b}$ Corresponding Author: Marco Cascella, Division of Anesthesiology, Department of Anesthesiology, Endoscopy and Cardiology, Istituto Nazionale Tumori Fondazione Pascale, Via Mariano Semmola, Naples I-80131, Italy. Email: m.cascella@istitutotumori.na.it

doi: http://dx.doi.org/10.14740/jocmr2362w are reported [3], PE secondary to upper limb SVT (without UEDVT) is a very rare clinical manifestation with few cases reported in the literature $[4,5]$.

We report a rare case of thrombophlebitis in departure from a superficial branch of the cephalic vein of the right arm, complicated by cardiac arrest secondary to a massive PE in a patient who underwent major surgery for ovarian cancer.

\section{Case Report}

A Caucasian female patient aged 48 years, with normal body mass index (weight $60 \mathrm{~kg}$ ), underwent bilateral hysteroannessectomy with omentectomy and lymphadenectomy for ovarian cancer. Standard deep venous thrombosis prophylaxis with low molecular weight heparin (Nadroparine 2850 Anti Xa IU sc) was employed. After the operation, the patient was transferred to the post-anesthesia care unit (PACU). Postoperative course in the first $24 \mathrm{~h}$ was normal, without any dyspnea, tachypnea, or hemodynamic changes. $\mathrm{PaO}_{2} / \mathrm{FiO}_{2}$ was normal.

After $24 \mathrm{~h}$, during the mobilization maneuvers for the discharge from PACU, the patient had a sudden loss of consciousness with cardiac arrest at the monitor. We started cardiopulmonary resuscitation (CPR). After $15 \mathrm{~min}$ of CPR, the patient had a pulseless electrical activity (PEA). However, at Doppler echocardiography, there was a residual contractile activity. After another $20 \mathrm{~min}$ of CPR pulse, consciousness reappeared.

In advanced cardiovascular life support, we treated a severe hypotension with both norepinephrine and dobutamine, climbing the dobutamine by 6 to $3 \mu \mathrm{g} / \mathrm{kg} / \mathrm{min}$ and increasing norepinephrine up to $0.14 \mu \mathrm{g} / \mathrm{kg} / \mathrm{min}$. In this phase, the Doppler echocardiography showed a clear right atrial dilatation. Which was the cause of PEA? The pulmonary CT angiography showed a massive bilateral PE (Fig. 1). In view of the recent and challenging surgery and the very low levels of hemoglobin $(6.7 \mathrm{~g} / \mathrm{dL})$ in association with the loss of approximately 700 $\mathrm{mL}$ of frankly blood material from surgical drains in $24 \mathrm{~h}$, it was decided not to practice thrombolysis. We organized the transfer in a highly specialized structure to operate the percutaneous fragmentation of the embolus. We started treatment with unfractionated heparin IV bolus of $80 \mathrm{IU} / \mathrm{kg}$ followed by an infusion of $18 \mathrm{IU} / \mathrm{kg} / \mathrm{h}$ with dose adjustments according to aprothrombin time. We simultaneously started the anticoagulant therapy with warfarin. The response to therapy was surprising. 


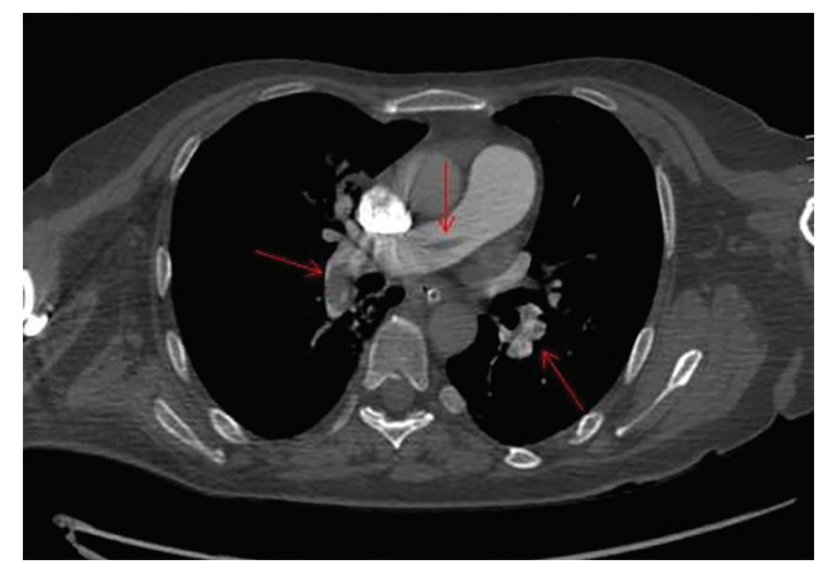

Figure 1. Pulmonary CT angiography axial showing multiple filling defects due to pulmonary artery embolism.

In about $3 \mathrm{~h}$, the pressure came back to acceptable values and the peripheral perfusion improved. Consequently, we desisted from making the planned percutaneous fragmentation.

Twenty-four hours later, the breath was only slightly tachypneic, there was still hypoxia but it had significant improvement $\left(\mathrm{PaO}_{2} / \mathrm{FiO}_{2}\right.$ indicative of moderate hypoxemia). Because the hemodynamic status was characterized by normal pressure values with slightly tachycardia, norepinephrine was gradually climbed. Meanwhile, the arm of the infusion (side cannulation of a superficial vein with a 16 Gouge catheter) and seat of the non-invasive monitoring of blood pressure (right arm) became swollen and painful. The subsequent color-Doppler ultrasound showed an extensive thrombosis of the right cephalic vein with absence of flow signals (Fig. 2). The thrombus did not extend to the axillary vein. Deep veins of the upper limbs, as well as the veins of the lower limbs, were all patent, without any sign of UEDVT or lower extremity deep venous thrombosis (LEDVT). We have researched additional conditions predisposing to thrombosis but there were no abnormality in the levels of homocysteine, as well as protein $\mathrm{C}$ and protein $\mathrm{S}$ deficiency.

Ten days after the acute episode, a new color-Doppler survey confirmed the thrombotic obliteration of the right cephalic vein with the presence of flow and patency in both the deep veins of the arm as well as the deep veins of the lower limbs. The arm became clinically deflated with clear signs of thrombophlebitis, with very hard cords along the course of the superficial veins.

\section{Discussion}

SVT is characterized by the combination of thrombosis and inflammation in a superficial vein. It involves the great saphenous vein in more than $60-80 \%$ of cases, the small saphenous vein in $10-20 \%$ of cases and much less frequently the veins of the upper limbs. It seems to be a higher prevalence in women and its incidence increases with age in both sexes [6]. Varicose veins are the most common predisposing factor, but there is a wide range of conditions that have been outlined, such as the

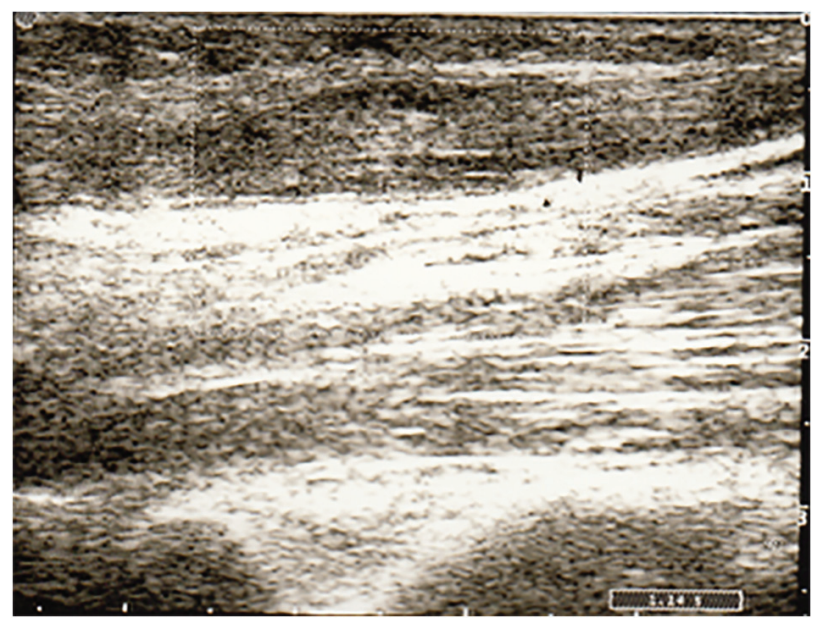

Figure 2. Color-Doppler scan of the right arm showing an enlarged cephalic vein, with echoic material in the lumen and absence of flow signals.

prolonged immobilization, trauma, obesity, and thrombophilic abnormalities of hemostasis, oral contraceptive or hormonal therapy, the previous history of LEDVT, UEDVT or SVT, the use of an intravenous catheter, malignancies and autoimmune disorders [7]. The pathophysiology of SVT can be explained in terms of external trauma, direct endothelial trauma, internal inflammation of the vein wall and alterations in hemostasis. While the external trauma can result from a direct external force (e.g. for compression by externally applied dressings), the internal trauma involves a direct endothelial damage (e.g. for a lesion by venous cannulation or for a continuous IV infusion). In both cases, the superficial vein exposed to injury produces edema and activation of leukocytes that predispose to thrombosis [8]. Thrombophlebitis is the most frequent complication of peripheral venous infusion and its most important predictor is the duration of catheterization [9]; the material and the dimensions of the catheter can affect the risk. According to Campbell [10] compared to small caliber catheters, the large bore catheters are associated with an increased risk, and the polyurethane catheters (PEU) have been associated with a reduction of $30-45 \%$ of the incidence of peripheral vein infusion thrombophlebitis compared to tetrafluoroethylenehexafluoropropylene (Teflon) catheters. The characteristics of the solutions for intravenous administration affect the occurrence of thrombophlebitis: high osmolality solutions and low $\mathrm{pH}$, such as glucose, conferring a higher risk [11]. Also, some drugs administered intravenously, such as potassium chloride, barbiturates, phenytoin, many chemotherapeutic agents and certain antibiotics (e.g. vancomycin, amphotericin B, and blactam antibiotics), have been associated with a doubled risk [12]. Another risk factor for SVT is the catheter infection: a percentage between $5 \%$ and $25 \%$ of peripheral catheters is colonized at the time of removal from the skin and the organisms colonized catheters are six times more likely to be associated with thrombophlebitis [13].

It remains to clarify the pathogenetic chain of events which in our case led to the considerable extension of the peripheral venous thrombosis and the massive pulmonary involvement. 
Probably it was the result of a synergy with enhancement of multiple risk factors: female gender, risk factors related catheter, intermittent compression of the cuff for measuring blood pressure. These risk factors have impacted the cephalic vein which is of small caliber, tortuous and full of valves and a system to $90^{\circ}$ in the axillary vein. Not surprisingly, the axillary vein is not the first choice for placement of PICCs and Medline.

An important consideration on the pathogenesis of thromboembolism is that the case report concerns a cancer patient and then in itself already at high risk of thrombosis. It is well known that the cancer diseases increase the risk of venous thromboembolism (VTE) [14-17] and about 20\% of cancer patients undergo thromboembolic events [16]. PE is the most common cause of postoperative mortality in cancer patients and is among the leading causes of death in patients with cancer [17]. In addition, gynecological surgery, even in the absence of malignancy, results in increased risk of VTE than the general surgery. Because our patient underwent to a complex surgery for ovarian cancer, she meet a higher risk for VTE.

Compression ultrasound and color-Doppler ultrasound allow detecting the venous thrombosis to assess its extent and to monitorize the thrombus evolution during the therapy [18, 19]. Nowadays, ultrasound represents the standard imaging modality and there is no diagnostic role left for phlebography [4]. Since the lower limbs represent the most frequent source of PE, usually lower extremity veins represent the first anatomical area to be investigated. In case of negative findings, the ultrasound examination is extended to the abdominal and pelvic veins as well as to the upper limbs veins. In our case, the clinical findings at level of the right upper limb, which became swollen and warm, directly drove our attention to the upper extremities.

Because data suggest that asymptomatic PE occurs up to a third of patients with DVT [20], we must assume that asymptomatic PE can occur even combined with SVT. Verlato et al [21] demonstrated a high rate of PE in patients with thrombophlebitis of the greater saphenous vein.

For these reasons, it is mandatory to stress prophylaxis of SVT in high-risk patients, such as those with cancer undergoing surgery. Prophylaxis of thrombosis is recommended for all patients admitted in oncology, with particular attention to cancer patients at high risk of thrombosis and those submitted or to be submitted to surgery [22]. Much attention should be paid to specific risk factors such as the infusion of solutions and drugs potentially harmful for the endothelium, as well as for the correct management of venous catheterization. About the changing of the peripheral catheter, a recent review found no evidence to support changing catheters every 72 - 96 h, suggesting that the insertion site should be inspected at each shift change and the catheter removed if signs of inflammation, infiltration, or blockage are present [23].

Another feature of our case was its rare as well as severe clinical manifestation: the SVT of the upper limbs became more complicated with a clinical picture of massive PE, best defined as PE with hemodynamic instability. This is a very rare report; in 1990 Sassu et al [4] reported a case of a patient with recurrent superficial thrombophlebitis of the left arm that developed right-sided $\mathrm{PE}$, but not massive PE. Although Barros et al [5] described a case of post-trauma superficial thrombophlebitis of the basilic vein complicated with PE, also in this case, the PE was not massive, but limited in the basal posterior and lateral segments of the right inferior lobe.

\section{Conclusion}

Clinicians are especially careful to prevention of embolism from LEDVT, while they often underestimated the UEDVT and SVT of the upper limb. Thanks to advances in the understanding of the pathophysiology of the SVT and the chance to make an early diagnosis, this complication although rare should be appropriately prevented, especially in cancer patients at high risk thrombosis, and its diagnosis for suspected serious complications can result. Our case is an example of the summation of numerous thrombotic risk factors simultaneously present, triggering a cascade of reactions and resulting in a potential fatal clinical manifestation.

\section{Conflicts of Interest}

The authors declare no conflict of interest.

\section{References}

1. Klitfod L, Broholm R, Baekgaard N. Deep venous thrombosis of the upper extremity. A review. Int Angiol. 2013;32(5):447-452.

2. Czihal M, Hoffmann U. Upper extremity deep venous thrombosis. Vasc Med. 2011;16(3):191-202.

3. Kesteven P, Robinson B. Superficial thrombophlebitis followed by pulmonary embolism. J R Soc Med. 2001;94(4):186-187.

4. Sassu GP, Chisholm CD, Howell JM, Huang E. A rare etiology for pulmonary embolism: basilic vein thrombosis. J Emerg Med. 1990;8(1):45-49.

5. Barros FS, Sandri JL, Prezotti BB, Nofa DP, Salles Cunha $\mathrm{SX}$, Barros SD. Pulmonary embolism in a rare association to a floating thrombus detected by ultrasound in the basilic vein at the distal arm. Rev bras ecocardiogr imagem cardiovasc. 2011;24(4):89-92.

6. Marchiori A, Mosena L, Prandoni P. Superficial vein thrombosis: risk factors, diagnosis, and treatment. Semin Thromb Hemost. 2006;32(7):737-743.

7. Leon L, Giannoukas AD, Dodd D, Chan P, Labropoulos N. Clinical significance of superficial vein thrombosis. Eur J Vasc Endovasc Surg. 2005;29(1):10-17.

8. Browse NL, Burnand KG, Thomas ML. Diseases of the veins: pathology, diagnosis and treatment. London: Hodder and Stoughton; 1988.

9. Lewis GB, Hecker JF. Infusion thrombophlebitis. Br J Anaesth. 1985;57(2):220-233.

10. Campbell L. IV-related phlebitis, complications and length of hospital stay: 1. Br J Nurs. 1998;7:1305-1311.

11. Lederle FA, Parenti CM, Berskow LC, Ellingson KJ. The idle intravenous catheter. Ann Intern Med. 
1992;116(9):737-738.

12. Monreal M, Quilez F, Rey-Joly C, Rodriguez S, Sopena N, Neira C, Roca J. Infusion phlebitis in patients with acute pneumonia: a prospective study. Chest. 1999;115(6):1576-1580.

13. Stratton CW. Infection related to intravenous infusions. Heart Lung. 1982;11(2):123-137.

14. Elyamany G, Alzahrani AM, Bukhary E. Cancer-associated thrombosis: an overview. Clin Med Insights Oncol. 2014;8:129-137.

15. Khorana AA. Cancer-associated thrombosis: updates and controversies. Hematology Am Soc Hematol Educ Program. 2012;2012:626-630.

16. Behranwala KA, Williamson RC. Cancer-associated venous thrombosis in the surgical setting. Ann Surg. 2009;249(3):366-375.

17. Khorana AA. Venous thromboembolism and prognosis in cancer. Thromb Res. 2010;125(6):490-493.

18. Blaivas M. Ultrasound in the detection of venous thromboembolism. Crit Care Med. 2007;35(5 Suppl):S224-234.

19. Gaitini D, Beck-Razi N, Haim N, Brenner B. Preva- lence of upper extremity deep venous thrombosis diagnosed by color Doppler duplex sonography in cancer patients with central venous catheters. J Ultrasound Med. 2006;25(10):1297-1303.

20. Stein PD, Matta F, Musani MH, Diaczok B. Silent pulmonary embolism in patients with deep venous thrombosis: a systematic review. Am J Med. 2010;123(5):426-431.

21. Verlato F, Zucchetta P, Prandoni P, Camporese G, Marzola MC, Salmistraro G, Bui F, et al. An unexpectedly high rate of pulmonary embolism in patients with superficial thrombophlebitis of the thigh. J Vasc Surg. 1999;30(6):1113-1115.

22. Farge D, Debourdeau P, Beckers M, Baglin C, Bauersachs RM, Brenner B, Brilhante D, et al. International clinical practice guidelines for the treatment and prophylaxis of venous thromboembolism in patients with cancer. J Thromb Haemost. 2013;11(1):56-70.

23. Webster J, Osborne S, Rickard CM, New K. Clinicallyindicated replacement versus routine replacement of peripheral venous catheters. Cochrane Database Syst Rev. 2013;4:CD007798. 